\title{
eJRIEPS
}

Ejournal de la recherche sur l'intervention en éducation physique et sport

12 | 2007

Varia

\section{Amélioration de la qualité de l'animation dans les activités de Québec en forme}

Sylvie Beaudoin, Jean-Pierre Brunelle et Carlo Spallanzan

\section{OpenEdition}

Journals

Édition électronique

URL : https://journals.openedition.org/ejrieps/6135

DOI : 10.4000/ejrieps.6135

ISSN : 2105-0821

Éditeur

ELLIADD

\section{Référence électronique}

Sylvie Beaudoin, Jean-Pierre Brunelle et Carlo Spallanzan, « Amélioration de la qualité de l'animation dans les activités de Québec en forme », eJRIEPS [En ligne], 12 | 2007, mis en ligne le 01 juillet 2007, consulté le 28 juin 2021. URL : http://journals.openedition.org/ejrieps/6135; DOI : https://doi.org/ 10.4000/ejrieps. 6135

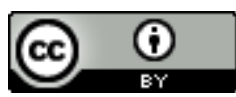

La revue eJRIEPS est mise à disposition selon les termes de la Creative Commons Attribution 4.0 International License. 
Sylvie Beaudoin ${ }^{1}$, Jean-Pierre Brunelle ${ }^{1}$ \& Carlo Spallanzani ${ }^{1-2}$

Faculté d'Éducation Physique et Sportive. Université de Sherbrooke, Sherbrooke, Canada ${ }^{1}$ GRIEFPAP; ${ }^{2}$ CRIE-CRIFPE

\section{Résumé*}

Depuis 2002, l'organisme Québec en forme vise, par la pratique de l'activité physique, le développement global de plus de 32000 enfants de milieux économiquement défavorisés dans l'ensemble de la Province de Québec au Canada. La préoccupation de bien répondre à la mission qu'elle s'est donnée incite l'organisme à se questionner sur les pratiques d'animation de ses activités physiques. À la suite de l'identification des besoins de formation, le modèle de la supervision clinique est utilisé pour permettre la prise en compte des difficultés rencontrées lors de l'intervention en milieu réel. Les résultats obtenus auprès d'une dyade d'animateurs sélectionnés démontrent que la formation a influencé leur pratique au point d'améliorer le temps de développement moteur offert au groupe. Toutefois, il a été nécessaire d'implanter la supervision clinique pour que le temps d'engagement moteur individuel s'améliore. La stratégie de formation et de supervision développée dans le cadre de cette recherche action collaborative sera utilisée comme unité de base pour la formation des formateurs de Québec en forme au niveau provincial.

\section{La problématique et le cadre de référence}

Les effets bénéfiques de la pratique régulière d'activité physique sont bien documentés dans la littérature (entre autres: Dwyer et al., 1983; Gruber, 1986; Alpert \& Wilmore, 1994; Sallis et al., 1997, 1999; Kino-Québec, 2000). Bien entendu, le jeune âge est une période critique pour l'adoption de valeurs prônant un mode de vie actif. De par sa nature, l'activité physique permet aux enfants d'interagir ensemble, de prendre des décisions et tout simplement, d'éprouver du plaisir (Martinek, Schilling \& Johnson, 2001; Hellison, 2003). Au Québec, plusieurs institutions, comme les organismes communautaires, les institutions scolaires, les différents paliers de gouvernement se préoccupent d'offrir aux enfants l'encadrement nécessaire à la pratique de l'activité physique. Par contre, les

\footnotetext{
* Le projet AQUA bénéficie du support financier de l'organisme Québec en forme (2004-2007).
} 
enfants qui vivent dans les milieux économiquement défavorisés bénéficient rarement des mêmes services et infrastructures que ceux qui vivent dans des communautés mieux nanties. Par conséquent, les enfants de ces milieux seraient moins actifs physiquement et plus sédentaires que ceux qui vivent dans des familles plus aisées (McGrath, Matthews \& Brady, 2006). Parallèlement, les conditions mises en place dans les programmes d'activité physique ne favorisent pas toujours la participation active des enfants. Bien souvent, les animateurs ont peu d'expérience en intervention sportive, n'ont pas suivi de formation académique reliée à ce champ d'activité et bénéficient rarement d'une supervision adéquate.

Depuis 2002, un organisme à but non lucratif, Québec en forme, s'est donné pour mission de contribuer à l'amélioration de la santé et de l'autonomie globale des enfants de quatre à 12 ans issus de milieux économiquement défavorisés. Cet organisme soutient l'implantation de programmes durables de participation à des activités physiques et sportives au sein des communautés locales $(n=25)$ de la Province de Québec et touche annuellement plus de 32000 enfants sur l'ensemble du territoire québécois (Québec en forme, 2003). La préoccupation de bien répondre à sa mission incite l'organisme à se questionner sur les pratiques d'animation de ses activités. Par le biais d'une recherche action, Québec en forme vise le développement d'une stratégie de formation et de supervision adaptée à sa clientèle d'animateurs. Le projet AQUA I (Amélioration de la Qualité de l'Animation) a été mis sur pied par le professeur Jean-Pierre Brunelle, Ph.D., de l'Université de Sherbrooke, en collaboration avec l'organisme Québec en forme et son directeur de la recherche, formation et évaluation, monsieur Jean-Guy Ouellet, Ph.D. Cette première formation, qui s'inscrit dans un programme à quatre niveaux, vise principalement à favoriser une participation motrice efficace des enfants en offrant des activités physiques dynamiques et sécuritaires, réalisées dans un contexte social stimulant.

Le projet AQUA I s'inscrit dans le courant de recherche sur l'efficacité de l'enseignement en éducation physique. En 1974, Dunkin et Biddle ont présenté la complexité des interactions possibles entre les variables de présage, de contexte, de processus et de produit dans leur modèle pour l'étude de l'enseignement. Vers la fin des années 1970, les travaux menés portaient sur l'élaboration d'une méthode d'évaluation de l'efficacité de l'enseignement sans avoir recours à la variable de processus. À cette époque, Siedentop et ses collègues ont mis sur pied une stratégie pour observer et analyser le travail des enseignants en éducation physique (Tousignant et Brunelle, 1982). Ce système d'analyse, l'Academic Learning Time in Physical Education (ALT-PE), consiste à mesurer 
le temps d'engagement efficace des participants dans les diverses activités proposées lors de la séance (Brunelle et al., 1996; Brunelle, 2002). En 1996, Brunelle et ses collègues ont proposé une troisième version française de l'ALT-PE, le Système d'Analyse du Temps d'Apprentissage (SATA). Ce dernier permet d'apprécier l'efficacité d'intervention par l'observation systématique des comportements des participants.

La première étape du projet AQUA I, essentielle à la compréhension du contexte d'intervention spécifique à Québec en forme, implique la description des variables de présage, de contexte et de processus telles que définies par Dunkin \& Biddle (1974). La réalisation d'une phase exploratoire en milieu réel permet de dresser cet état des lieux nécessaire au développement d'une stratégie de formation et de supervision adaptée aux besoins. Néanmoins, parmi l'ensemble des variables constitutives d'une intervention en activité physique, nous avons retenu celles qui nous semblaient les plus représentatives pour apprécier l'efficacité de l'animation. En se basant sur les résultats de plusieurs recherches sur l'efficacité de l'enseignement en éducation physique (Brunelle et al., 1996), sur la mission de Québec en forme, ainsi que sur des observations préalables et des besoins exprimés par les intervenants locaux, l'approche privilégiée consiste à utiliser le temps de développement moteur offert au groupe et le temps d'engagement moteur individuel comme variables permettant de porter un jugement sur l'efficacité de l'animation.

De plus, s'inspirant de l'approche développementale de l'acte pédagogique de l'intervenant (Brunelle et Brunelle, 2002), le projet AQUA I utilise le cadre de référence de la supervision pédagogique comme stratégie pour accompagner le changement. Ce type de supervision consiste en l'établissement d'une relation d'aide entre un superviseur et un supervisé, en ayant comme objectif l'amélioration de la pratique pédagogique (Brunelle et al., 1988, Brunelle et al., 1991). La diversité du contexte de Québec en forme nous amène à croire qu'une approche de supervision clinique, qui met l'accent sur la résolution de problèmes concrets vécus par le supervisé au sein d'un processus de collaboration (Brunelle et al., 1988, 1991), serait la plus appropriée dans le cadre du projet AQUA I. Ce modèle semble en effet être le mieux adapté pour favoriser l'autonomie des animateurs tout en leur offrant, par le processus de supervision, le support nécessaire à leur démarche.

La conduite d'un processus de supervision clinique suppose au préalable le développement d'un lien de confiance entre le superviseur et le supervisé, prémisse nécessaire à une saine collaboration lors des rencontres de supervision. De façon 
opérationnelle, le processus de supervision clinique élaboré dans le cadre du projet AQUA I se présente comme suit. Lors de la première rencontre, le superviseur et le supervisé élaborent conjointement l'objet de la supervision et les objectifs à atteindre lors de la séance. Par la suite, le superviseur assiste, sur place, à la séance d'animation pilotée par le supervisé. II recueille tout d'abord des données sur le comportement des enfants, soit le temps de développement moteur offert au groupe (DM) et le temps d'engagement moteur individuel (TEM). Parallèlement, il utilise le cadre de référence proposé par Brunelle et Brunelle (2002) pour relever des exemples et des contreexemples de compétences de base en intervention maîtrisées ou non par l'animateur. Les compétences de base s'insèrent au sein de quatre capacités générales, soit de gérer efficacement (organiser le groupe et le matériel, gérer les transitions, prévenir et gérer les comportements perturbateurs), de communiquer intelligiblement (expliquer clairement par le langage oral et non verbal, s'exprimer de manière audible et motivante), d'établir des relations motivantes (gérer l'accueil et le bilan de fin de séance, se positionner pour observer et écouter, manifester de l'enthousiasme et de l'ouverture envers les enfants) et finalement, de faire apprendre (présenter les contenus de la séance, adapter le degré de difficulté des exercices aux capacités des enfants, gérer la répartition et la justesse des rétroactions, intégrer les apprentissages en fin de séance) (Desbiens, J.-F., Brunelle, J.P., Spallanzani, C. et Roy, M., 2005). Ces compétences de base servent de trame de fond à l'élaboration d'hypothèses d'action lors des rencontres de supervision. Immédiatement après la séance, le superviseur analyse les données recueillies et apprécie l'atteinte des objectifs fixés en rencontre pré-séance. La rencontre de supervision, réalisée sur place suite à la séance, permet au superviseur de présenter les résultats au supervisé et de discuter avec lui de l'atteinte des objectifs fixés au départ. Le développement de nouvelles hypothèses d'action, basées sur les constats relevés quant à la maîtrise des compétences de base, conduit à l'initiation d'un nouveau cycle de supervision. La figure 1 résume les phases du processus de supervision clinique mis en place dans le cadre du projet AQUA I. 


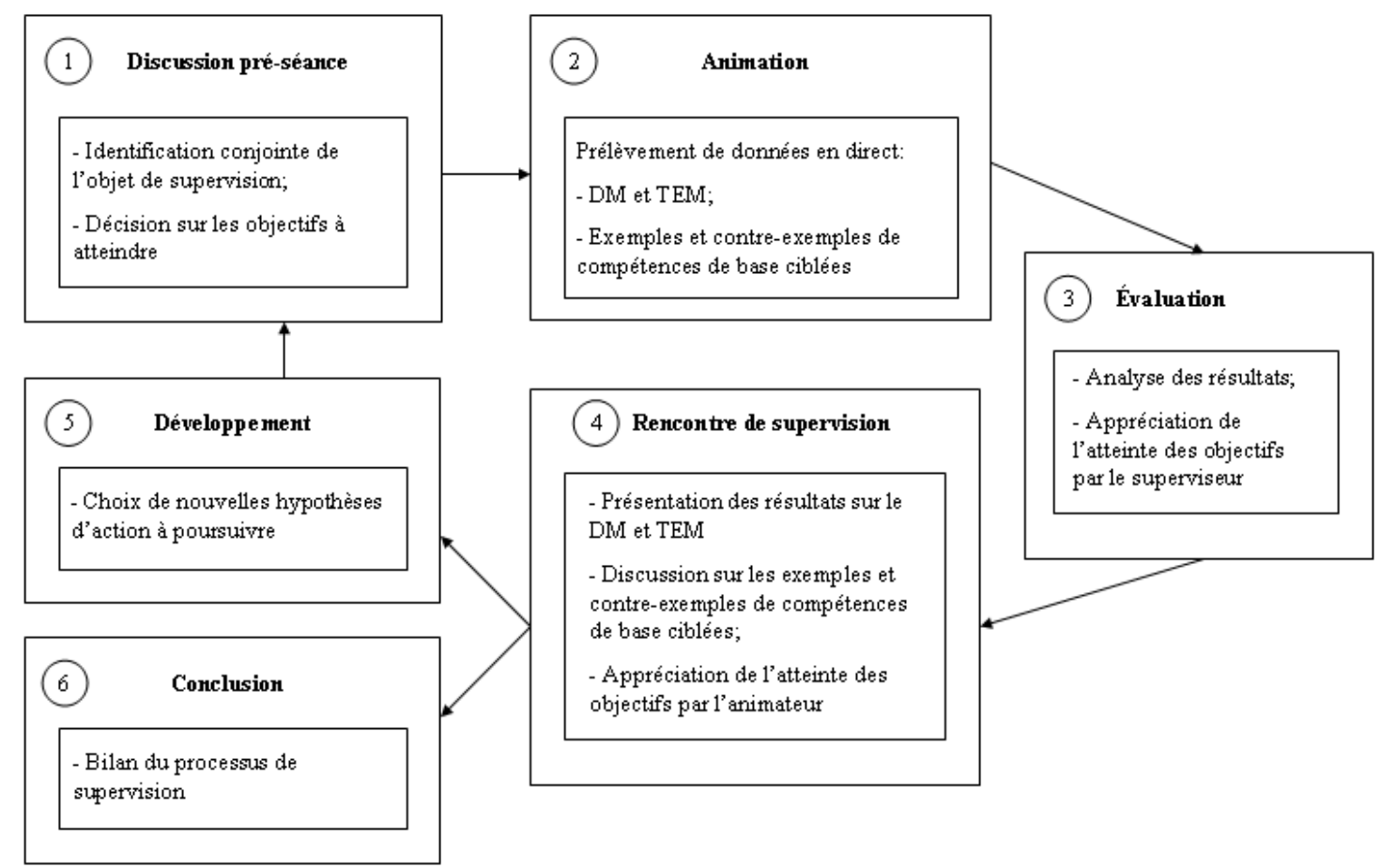

Figure 1: Les phases d'un processus de supervision clinique

Adapté de Brunelle, J., Drouin, D., Godbout, P. et Tousignant, M. (1988). La supervision de l'intervention en activité physique. Montréal : Gaëtan Morin Éditeur, p. 20.

\section{Les objectifs}

L'approche novatrice du projet AQUA cherche à provoquer un changement au niveau des pratiques d'animation d'activités physiques. L'objectif général du niveau I est de favoriser une participation motrice efficace des enfants en offrant des activités physiques dynamiques et sécuritaires, réalisées dans un contexte social stimulant. L'objectif spécifique de la présente étude consiste à déterminer les besoins de formation et de supervision des animateurs de Québec en forme, pour améliorer le temps d'engagement offert aux enfants qui leur sont confiés. Cette recherche action collaborative repose sur le développement d'une stratégie de formation et de supervision auprès d'une première cohorte d'animateurs de l'organisme Québec en forme.

\section{La méthodologie}

Le projet vise l'implantation d'un processus de formation et de supervision dans une région qui participe au programme de Québec en forme. Neuf animateurs se sont portés 
volontaires pour participer à cette étude. Ces animateurs sont âgés d'environ 28 ans et interviennent en moyenne une vingtaine d'heures par semaine dans les activités de Québec en forme. La majorité a obtenu ou est en voie d'obtenir un diplôme universitaire, la plupart dans un autre domaine que l'intervention en activité physique. L'expérience en animation de ces animateurs varie de quelques mois à 14 ans.

Une stratégie de recherche action nous est apparue la plus pertinente pour ce genre d'étude. De par sa méthodologie adaptée aux besoins du milieu, la recherche action constitue «une façon opérationnelle de mieux comprendre la complexité de l'intervention dans un milieu de travail et d'y apporter des modifications» (Brunelle et al., 1988, p.13). De même, la recherche action collaborative sous-entend qu'il y a une «inter influence entre la pratique et la recherche», le chercheur visant une «production de connaissances qui inclue et tienne compte du point de vue du praticien et des contraintes de son contexte d'action» (Desgagné et al., 2001, p.39). De plus, l'étude est menée selon un protocole à cas unique qui permet de voir, à partir d'observations systématiques, l'évolution des sujets en fonction des changements attendus (Ladouceur \& Bégin, 1980).

La méthodologie du projet AQUA I, élaborée en fonction des caractéristiques du milieu, se déroule en trois phases. Une phase exploratoire est tout d'abord effectuée pour établir le niveau de base du comportement des enfants durant les conditions de réalisation. Par la suite, une formation est donnée aux animateurs pour provoquer un changement au niveau des pratiques d'animation. Finalement, un suivi est effectué afin de vérifier la stabilité ou le changement au niveau du comportement des enfants. La figure 2 présente les trois phases du projet AQUA I.

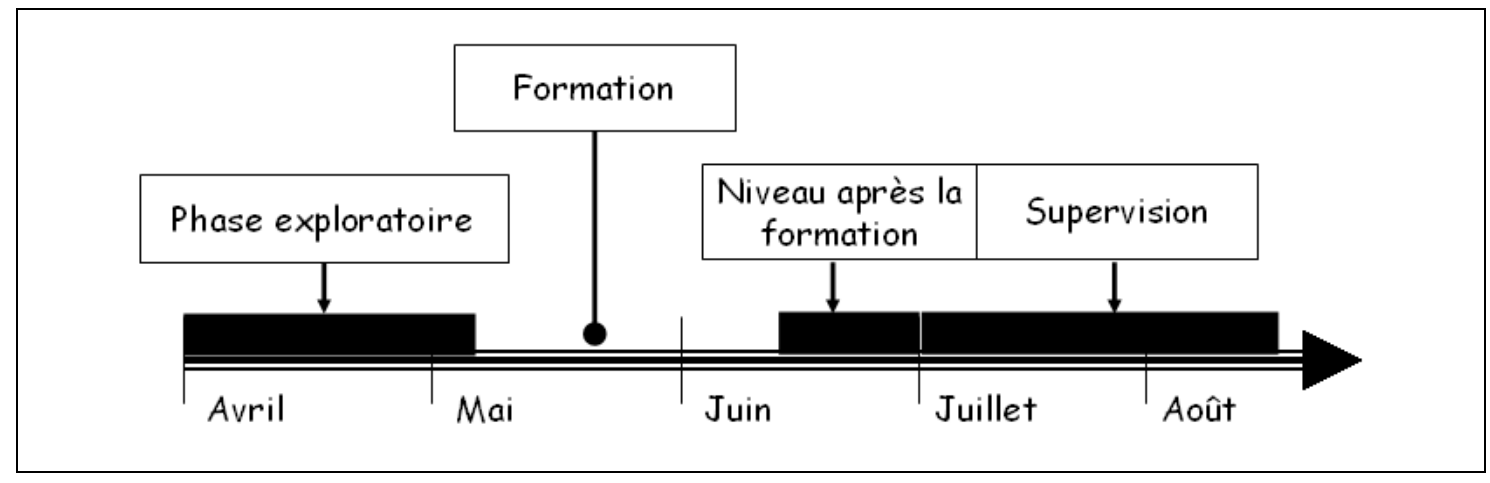

Figure 2 : Les trois phases du projet AQUA I 
3. 1. La phase exploratoire

De façon opérationnelle, la phase exploratoire implique l'observation directe de neuf séances d'animation d'activités physiques auprès des neuf animateurs volontaires. Le temps de développement moteur offert au groupe (DM) est mesuré à l'aide de l'enregistrement de la durée (Brunelle et al., 1988). Pour sa part, le temps consacré à l'engagement moteur individuel (TEM) est évalué à l'aide de la technique du balayage visuel à la fin d'un intervalle long, telle que présentée et validée par Brunelle et al. (1988). Le résultat obtenu au test de fidélité inter-analystes est de $89 \%$, ce qui assure la crédibilité de la cueillette de données.

\section{2. La formation}

Lors de la deuxième phase, une formation de groupe d'une durée de six heures est donnée par un chercheur universitaire à ces mêmes animateurs $(n=9)$. Cette activité a pour objectif de sensibiliser les animateurs à des pratiques d'animation efficaces. L'état des lieux effectué lors de la phase exploratoire permet de proposer aux animateurs un contenu pédagogique adapté à leurs besoins. Le thème général abordé lors de la formation est de favoriser une participation motrice efficace des enfants en offrant des activités physiques dynamiques et sécuritaires, réalisées dans un contexte social stimulant. L'accent est porté sur le temps d'engagement moteur et sur les conditions susceptibles de l'influencer.

\section{3. La supervision}

À la suite de la formation, une dyade d'animateurs qui co-animent ensemble, déjà observée lors de la phase exploratoire, s'est portée volontaire pour s'investir dans un processus de supervision. Au départ, trois séances d'animation $(n=3)$ sont observées sans rétroaction de notre part afin de vérifier l'intégration des contenus enseignés lors de la formation. À nouveau, le temps de développement moteur offert au groupe et le temps d'engagement moteur individuel sont mesurés.

Finalement, un processus de supervision est initié avec les deux co-animateurs. L'objectif visé est de les accompagner vers l'intégration des contenus enseignés lors de la formation afin de provoquer un changement au niveau de l'efficacité de l'animation. Le processus de supervision regroupe 12 observations en direct, en milieu naturel, immédiatement suivies de 12 rencontres de supervision.

La démarche scientifique suivie lors de la réalisation du projet AQUA I requiert la prise en compte de certaines précautions méthodologiques afin de s'assurer de la crédibilité des données recueillies (Brunelle, 1993). Tout d'abord, l'accessibilité au milieu est assurée par l'entremise des responsables de l'exécutif de Québec en forme. L'équipe de recherche a 
accès aux institutions scolaires et communautaires, aux horaires des activités et aux animateurs durant toute la durée du processus. Les animateurs sélectionnés doivent donner leur consentement éclairé sous forme écrite préalablement à toute participation au projet de recherche. La durée de la collecte de données, qui se déroule sur une période de cinq mois, ainsi que l'intensité des observations recueillies nous permet d'assurer une bonne compréhension de ce qui se passe sur le terrain. Finalement, l'exactitude des interprétations est vérifiée en utilisant différents moyens de collecte de données et en corroborant les informations recueillies auprès des animateurs.

\section{L'interprétation des résultats}

Rappelons tout d'abord que l'objectif général du projet AQUA I est de favoriser une participation motrice efficace des enfants en offrant des activités physiques dynamiques et sécuritaires, réalisées dans un contexte social stimulant. Plus spécifiquement, la présente étude vise à déterminer les besoins de formation et de supervision des animateurs de Québec en forme, pour améliorer le temps d'engagement moteur offert aux enfants qui leur sont confiés. Nous présentons maintenant les résultats obtenus lors de chacune des phases du projet AQUA I.

\section{1. La phase exploratoire}

Les observations effectuées lors de la phase exploratoire permettent de tracer un portrait de l'engagement moteur des enfants pendant les activités de Québec en forme. La figure 3 présente la compilation des résultats de neuf séances d'animation, pilotées par des animateurs différents, constituées de jeux d'animation et d'activités sportives. Ces activités poursuivent le même objectif, soit de favoriser une participation motrice efficace des enfants en offrant des activités physiques dynamiques et sécuritaires, réalisées dans un contexte social stimulant. La durée des séances d'animation observées varie de 30 à 90 minutes, pour une moyenne de 60 minutes. Les données quantitatives recueillies lors des observations sont transposées sur une unité de base de 60 minutes et présentées sous forme d'histogrammes comparatifs. Cette façon de faire permet de bien illustrer l'évolution de l'efficacité de l'animation aux différentes étapes de la stratégie de formation et de supervision. 


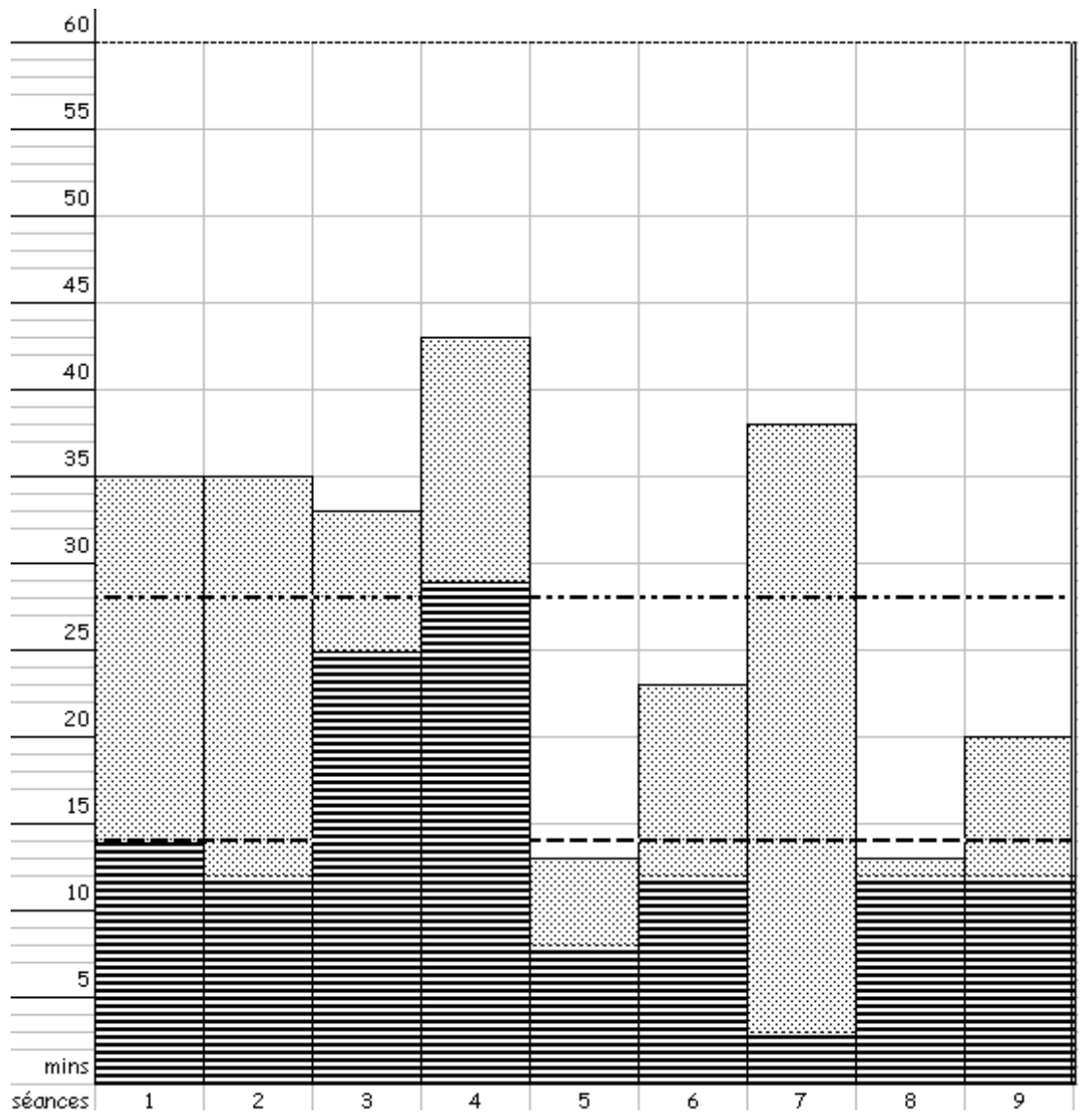

\begin{tabular}{|c|c|}
\hline \\
\hline \multirow{2}{*}{ (---- } & Légende \\
\hline & $\begin{array}{l}\text { Durée totale de la séance } \\
\text { Durée de développement moteur offert au groupe }\end{array}$ \\
\hline \multirow{3}{*}{ 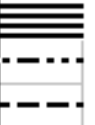 } & Durée d'engagement moteur individuel \\
\hline & Moyenne de la durée de développement moteur offe \\
\hline & Moyenne de la durée d'engagement moteur individue \\
\hline
\end{tabular}

Figure 3 . La compilation des résultats de neuf séances d'animation pilotées par des animateurs différents - phase exploratoire

De manière générale, le temps de développement moteur offert au groupe représente moins de la moitié du temps de la séance, soit 28 minutes. Quant au temps d'engagement moteur individuel, il représente une moyenne de 14 minutes par séance. De plus, l'écart type entre les différents animateurs est de dix minutes pour le temps de développement moteur offert au groupe et de sept minutes pour le temps d'engagement moteur individuel. Compte tenu du profil varié des animateurs observés, une piste d'interprétation de la variabilité des résultats pourrait être une utilisation plus ou moins grande d'activités d'exclusion et de modes d'organisation inefficaces, un faible degré d'expertise, ainsi qu'un manque de préparation de certains animateurs. Les résultats de la phase exploratoire sont comparables à ceux obtenus lors d'études antérieures en éducation physique, qui indiquent que l'engagement moteur de l'élève représente en moyenne de 15 à $20 \%$ de la 
durée d'une séance habituelle (Piéron, 1992). Selon cet auteur, « ce niveau d'engagement moteur est insuffisant pour exercer une action efficace sur le système cardio-respiratoire et produire des effets appréciables sur la santé » (p.108).

4. 2. La formation

À la suite de l'observation systématique et de l'analyse des activités proposées par Québec en forme, nous avons pu constater: 1) la faible quantité de développement moteur offert au groupe et 2) le faible taux d'engagement moteur individuel. Ces constats ont orienté une formation pédagogique de six heures, qui fut dispensée au groupe d'animateurs observés lors de la phase exploratoire. Parmi ces animateurs, une dyade s'est portée volontaire pour s'investir dans un processus de supervision actif et intensif. Quelques semaines après la formation, trois séances d'animation pilotées par ces deux co-animateurs ont été observées sans faire de rétroaction. Les résultats présentés sont mis en relation avec ceux obtenus par ces mêmes co-animateurs avant la formation (figure 4). 


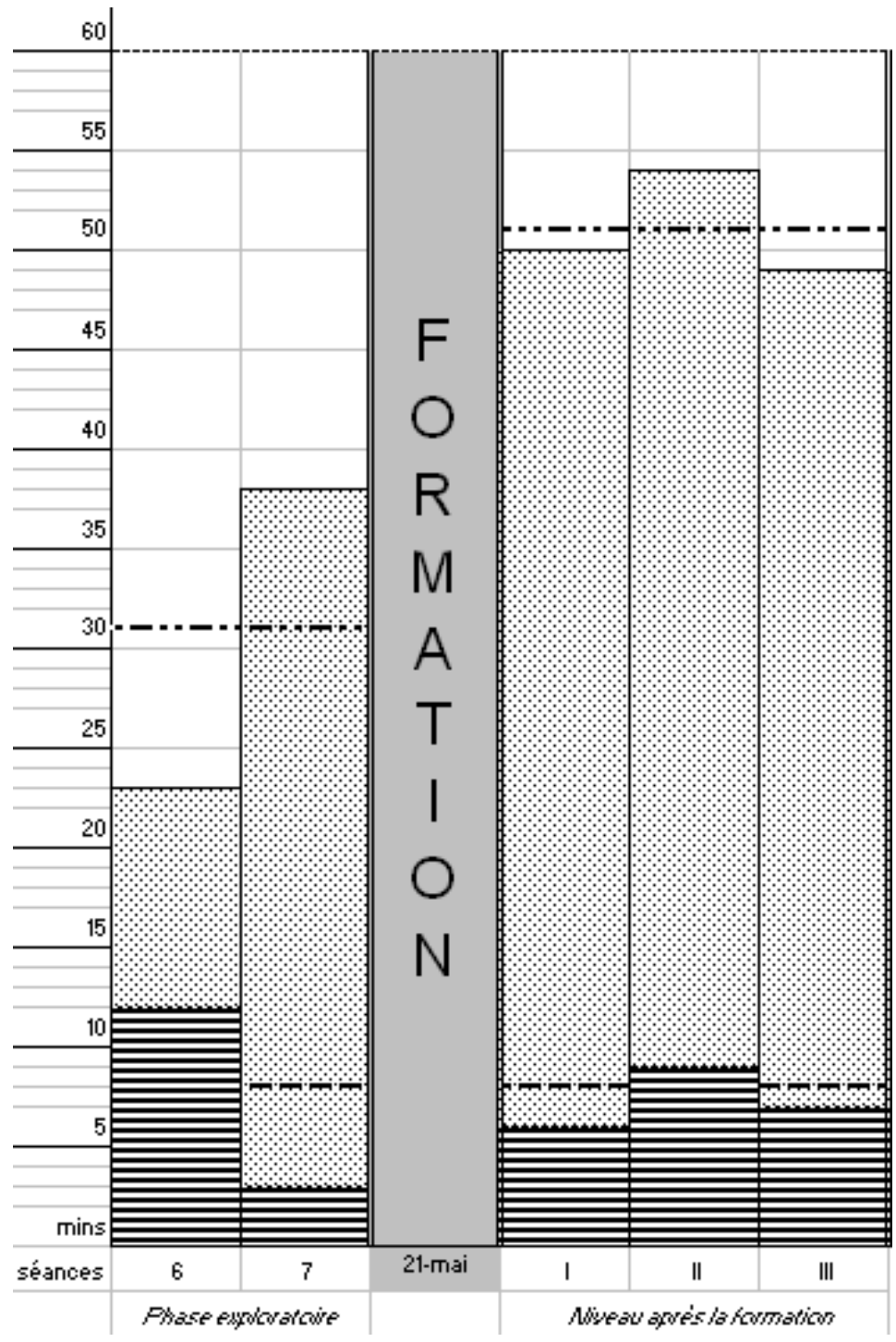

\begin{tabular}{|c|c|}
\hline \multicolumn{2}{|c|}{ Légende } \\
\hline & Durée totale de la séance \\
\hline & Durée de développement moteur offert au groupe \\
\hline & Durée d'engagement moteur individuel \\
\hline$-\cdots$ & Moyenne de la durée de développement moteur offert au groupe \\
\hline- - - & Moyenne de la durée d'engagement moteur individuel \\
\hline
\end{tabular}

Figure 4. Le temps d'engagement moteur avant et après la formation lors des séances pilotées par les co-animateurs

Ainsi, les résultats montrent un changement positif du temps de développement moteur offert au groupe suite à la formation. Les co-animateurs ont possiblement intégré certains éléments d'organisation efficace permettant une réduction du temps consacré aux situations préparatoires. Par contre, le temps d'engagement moteur individuel reste relativement stable et faible, avec une moyenne de sept minutes par séance. Les coanimateurs n'ont pas pu suffisamment opérationnaliser les notions portant sur l'amélioration du temps d'engagement moteur individuel enseignées lors de la formation. 


\section{3. La supervision}

Par la suite, un processus de supervision a été conduit avec cette même dyade d'animateurs. Cette démarche se compose de 12 observations systématiques en milieu réel et de 12 rencontres de supervision.
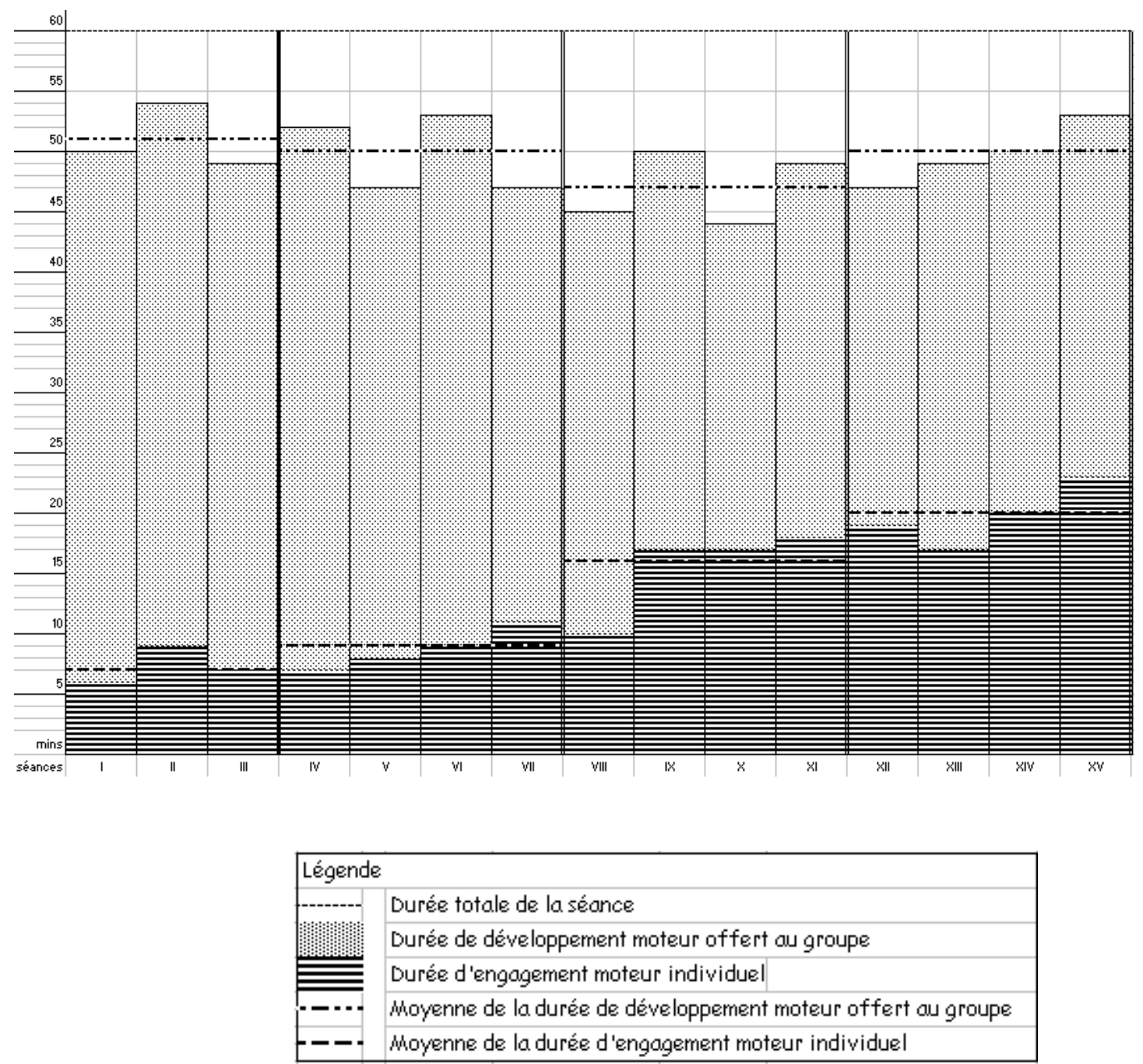

Figure 5. La progression du temps d'engagement moteur lors du processus de supervision

II est intéressant de constater une augmentation graduelle de l'engagement moteur individuel des enfants au fil des rencontres de supervision. En effet, la durée de l'activité motrice passe d'une moyenne de sept minutes lors de la mesure après la formation à près du triple, soit 20 minutes, à la fin du processus de supervision. L'analyse de contenu des rencontres de supervision démontre que la prise de conscience de l'engagement moteur des enfants s'est avérée être la trame de fond de chaque rencontre de supervision. De plus, les hypothèses d'action choisies tout au long du processus de supervision ont permis 
aux animateurs de graduellement améliorer la maîtrise de leurs compétences de base en intervention. De manière plus spécifique, les co-animateurs ont amélioré leur positionnement, leur balayage visuel et la mise en place de routines d'organisation, ce qui a conduit à des ajustements en cours de séance pour favoriser l'engagement moteur des enfants.

\section{Conclusion}

L'originalité de la présente étude repose sur l'établissement d'un rapport différent à la supervision. Au lieu de se centrer à modeler le comportement de l'animateur, il est proposé d'analyser l'effet de cette modification sur le comportement des enfants pendant les conditions de réalisation. Cet indicateur induit une perspective de supervision différente, qui amène le supervisé à se décentrer de lui-même pour porter son regard sur les enfants et l'activité. La forme de supervision proposée s'insère dans une approche globale tenant compte de la complexité de l'intervention en activité physique.

Il est révélateur de constater que les animateurs ont eu besoin d'un suivi soutenu et prolongé pour intégrer dans leurs interventions les concepts enseignés en formation. La conduite de cycles de supervision clinique a eu pour effet d'améliorer le temps d'engagement moteur individuel (TEM) des enfants au point de rejoindre la recommandation émise par Kino-Québec dans l'Avis du comité scientifique sur l'activité physique, déterminant de santé chez les jeunes (Kino-Québec, 2000). L'amélioration du temps d'engagement moteur reste un problème actuel au cœur de l'intervention en activité physique. L'inscription de cette préoccupation au sein de l'écologie globale du système reste un défi quotidien auquel font face les intervenants en activité physique. Nous sommes d'avis qu'une mobilisation corporelle efficace, réalisée dans un contexte social stimulant en compagnie d'un adulte signifiant, est nécessaire pour développer une attitude favorable face à la pratique de l'activité physique.

Au Québec, la forte croissance du programme Québec en forme témoigne d'une préoccupation grandissante envers la santé des enfants de milieux défavorisés. Une piste d'avenir intéressante pour la recherche en intervention en activité physique pourrait être de se questionner sur les catalyseurs du changement dans les milieux défavorisés en matière de pratique régulière d'activité physique. La connaissance des conditions qui prédisposent au changement permettrait la construction d'outils opérationnels pour accompagner les intervenants locaux dans la prise en charge de la qualité de l'animation de leurs activités et ainsi aider davantage les enfants dans leur développement. 


\section{Références}

Alpert, B.S. \& Wilmore, J.H. (1994). Physical activity and blood pressure in adolescents. Pediatric Exercise Science, 6, 361-380.

Brunelle, J. (2002). L'intervention en activité physique et sportive : un point de vue sur la recherche nord-américaine. Avante, 8 (2), 1-13.

Brunelle, J. \& Brunelle, J.P. (2002). L'observation et le feed-back dans une perspective de supervision pédagogique de stagiaires en enseignement de l'éducation physique et à la santé. Document de travail. Manuscrit non publié, Université de Sherbrooke, Faculté d'éducation physique et sportive, Sherbrooke, Québec.

Brunelle, J., Coulibaly, A., Brunelle, J.P., Martel, D. \& Spallanzani, C. (1991). La supervision pédagogique. Éducation physique et sport, 227, 58-63.

Brunelle, J., Drouin, D., Godbout, P. \& Tousignant, M. (1988). La supervision de l'intervention en activité physique. Montréal : Gaëtan Morin Éditeur.

Brunelle, J., Tousignant, M., Godbout, P., Spallanzani, C., Brunelle, J.P., Martel, D., Trudel, P., Gagnon, J., Savard, C. \& Rami, A. (1996). Le temps d'apprentissage. Ste-Foy : L'intervention éducative.

Brunelle, J.P. (1993). Le hockey éducatif. Contribution de la pratique du hockey compétitif au développement de la personne. Ste-Foy : L'intervention éducative.

Desbiens, J.F., Brunelle, J.P., Spallanzani, C. \& Roy, M. (2005). Former les personnes enseignantes associées (PEA) pour améliorer la supervision des stagiaires de la Faculté d'éducation physique et sportive (FEPS) de I'Université de Sherbrooke. Colloque 9 «Le développement professionnel des enseignants en contexte de changement: des interventions pour soutenir leurs apprentissages». Actes du $20^{\circ}$ congrès international de I'AIPU. (CDRom). Sherbrooke : Université de Sherbrooke, 27 au 30 mai 2003.

Desgagné, S., Bednarz, N., Lebuis, P., Poirier, L. \& Couture, C. (2001). L'approche collaborative de recherche en éducation : un rapport nouveau à établir entre recherche et formation. Revue des Sciences de l'Éducation, 27 (1), 33-65.

Dunkin, M.J. \& Biddle, B.J. (1974). The study of teaching. New York : Holt, Rinehart, and Winston.

Dwyer, T., Coonan, W.E., Leitch, D.R., Hetzel, B.S. \& Baghurst, R.A. (1983). An investigation of the effects of daily physical activity on the health of primary school students in South Australia. International Journal Epidemiology, 12 (3), 308-313. 
Gruber, J.J. (1986). Physical activity and self-esteem development in children: a metaanalysis. In G.A. Stull \& H.M. Eckert (Eds). Effects of physical activity on children. Champaign, IL : Human Kinetics.

Hellison, D. (2003). Teaching responsibility through physical activity. 2nd ed. Champaign, Illinois : Human Kinetics.

Kino-Québec (2000). L'activité physique, déterminant de la santé des jeunes, avis du comité scientifique de Kino-Québec. Secrétariat au loisir et au sport, ministère de la Santé et des Services sociaux, Gouvernement du Québec.

Ladouceur, R. \& Bégin, G. (1980). Protocoles de recherche en sciences appliquées et fondamentales. St-Hyacinthe : Édisem.

Martinek, T., Schilling, T. \& Johnson, D. (2001). Transferring personal and social responsibility of underserved youth to the classroom. The Urban Review, 33 (1), 29-45.

McGrath, J.J. Matthews, K.A., \& Brady, S. (2006). Individual versus neighbourhood socioeconomic status and race as predictors of adolescent ambulatory blood pressure and heart rate. Social Science and Medicine, 63, 1442-1453.

Piéron, M. (1992). Pédagogie des activités physiques et du sport. Paris : Éditions Revue EPS.

Québec en forme (2003). Rapport annuel 2002-2003. Document téléaccessible à l'adresse URL : <http://www.quebecenforme.org>.

Sallis, J.F., McKenzie, T.L., Alcaraz, J.E., Kolody, B., Faucette, N. \& Hovell, M.F. (1997). The effects of a two-year physical education program (SPARK) on physical activity and fitness in elementary school students. Sports, Plays and Active Recreation for Kids. American Journal of Public Health, 87 (8), 1328-1334.

Sallis, J.F., McKenzie, T.L., Kolody, B., Lewis, M., Marshall, S. \& Rosengard, P. (1999). Effects of health-related physical education on academic achievement: project SPARK. Research Quarterly for Exercise and Sport, 70, 127-134.

Tousignant, M. \& Brunelle, J. (1982). Les courants de recherche en enseignement de l'éducation physique. Revue des Sciences de l'Éducation, 8 (1), 63-77. 